\title{
Holographic Complexity and Thermodynamic Volume
}

\author{
Abdulrahim Al Balushi®, ${ }^{1, *}$ Robie A. Hennigar@ ${ }^{2, \dagger}$ Hari K. Kunduri®, ${ }^{2, \ddagger}$ and Robert B. Mann $\oplus^{1, \S}$ \\ ${ }^{1}$ Department of Physics and Astronomy, University of Waterloo, Waterloo, Ontario N2L 3G1, Canada \\ ${ }^{2}$ Department of Mathematics and Statistics, Memorial University of Newfoundland, St. John's, \\ Newfoundland and Labrador A1C 5S7, Canada
}

(Received 30 August 2020; revised 10 December 2020; accepted 10 February 2021; published 10 March 2021)

\begin{abstract}
We study the holographic complexity conjectures for rotating black holes, uncovering a relationship between the complexity of formation and the thermodynamic volume of the black hole. We suggest that it is the thermodynamic volume and not the entropy that controls the complexity of formation of large black holes in both the complexity equals action and complexity equals volume proposals in general. Our proposal reduces to known results involving the entropy in settings where the thermodynamic volume and entropy are not independent, but has broader scope. Assuming a conjectured inequality is obeyed by the thermodynamic volume, we establish that the complexity of formation is bounded from below by the entropy for large black holes.
\end{abstract}

DOI: 10.1103/PhysRevLett.126.101601

In recent years there has been dramatic progress in understanding the connections between gravity and quantum information. The quintessential example of this is entanglement in the context of the Anti-de Sitter/Conformal Field Theory correspondence (AdS/CFT) correspondence. Through the Ryu-Takayanagi prescription and its generalizations [1-4] the duality relates entanglement between spacetime regions in the field theory to the existence of minimal surfaces in the bulk, a situation often described by the slogan "entanglement = geometry."

Recently it has been suggested that entanglement may not be sufficient to fully describe physics in extreme regimes, such as the late-time dynamics of black holes $[5,6]$, and that instead complexity of a dual CFT state provides information that entanglement does not. Roughly speaking, complexity provides a measure of how difficult it is to construct certain states in the theory starting from simple unentangled states using a fixed set of universal gates. While well established in quantum mechanics, circuit complexity in quantum field theory is an area of active investigation, and there remains much to understand about its role in the holographic dictionary $[7,8]$. There have been a number of proposals suggesting how the complexity of the field theory state should be expressed in terms of bulk observables. The two most well studied of these proposals are the "complexity = volume" (CV) [9] and "complexity = action" (CA) $[10,11]$ conjectures. The

Published by the American Physical Society under the terms of the Creative Commons Attribution 4.0 International license. Further distribution of this work must maintain attribution to the author(s) and the published article's title, journal citation, and DOI. Funded by SCOAP ${ }^{3}$. former relates complexity to the volume of extremal codimension-one surfaces in the bulk, while the latter relates complexity to the value of the gravitational action on a region of spacetime known as the Wheeler-DeWitt (WDW) patch [12].

A number of properties of complexity as defined by the $\mathrm{CV}$ and CA proposals are now well-understood for black holes, with both proposals generally yielding qualitatively similar results, but not always [13-20]. For example, in both proposals it is known that at late times the complexity grows linearly in time at a rate characterized by the mass, or other thermodynamic potentials, of the black hole $[11,21-24]$. In both proposals the response of complexity to perturbations follows the "switchback effect" [16,25]. Most relevant for us here is the finding of Ref. [7] (see also Ref. [23]) that in both proposals the complexity of formation of large, (un)charged static, and spherically symmetric black holes is proportional to the black hole entropy.

Here we report on the first investigation of complexity for rotating black holes. From a holographic perspective, rotating black holes are dual to thermofield double states living on a rotating spacetime [26-28]. However, our main motivation here is to exploit the more complicated geometric structure of rotating black holes to test the complexity proposals for universal and divergent features that may not be evident in simpler geometries. The approach of understanding the behavior of an observable under deformations of the state or theory (e.g., through the addition of higher-curvature terms in the action) has been a fruitful line of investigation for identifying universal relationships and testing conjectures in the context of AdS/CFT [29-34].

We shall exploit the observation that the causal structure of a class of odd-dimensional rotating black holes is far simpler than the general situation. This allows for 
computations that would be effectively intractable in the general situation to be carried out largely analytically. Remarkably we find a connection between the complexity of formation and the thermodynamic volume $V$ of the black hole, indicating that it is this quantity and not the entropy that governs its behavior in both the $\mathrm{CV}$ and $\mathrm{CA}$ proposals. In the static limit, we recover previously known results.

Thermodynamic volume is a quantity that arises naturally when generalizing the Komar definition of mass from asymptotically flat spacetimes to those with (A)dS asymptotics and plays a central role in extending Smarr's formula from flat spacetimes to AdS spacetimes [35,36]. This extended Smarr relation reads

$$
(D-3) M=(D-2) T S+(D-2) \Omega_{i} J^{i}+\frac{\Lambda V}{4 \pi G_{N}},
$$

where $D$ is the spacetime dimension, $T$ is the Hawking temperature, $S$ is the entropy, $\Omega_{i}$ are the horizon angular velocities, $J^{i}$ are the independent angular momenta, $\Lambda \equiv$ $-(D-1)(D-2) / 2 \ell^{2}$ is the cosmological constant, and $\ell$ is the AdS length scale. If one allows for variations in the cosmological constant, the thermodynamic volume appears as the conjugate quantity to variations in $\Lambda$ :

$$
\delta M=T \delta S+\Omega_{i} \delta J^{i}-\frac{V \delta \Lambda}{8 \pi G_{N}} .
$$

Interpreting $P \equiv-\Lambda /\left(8 \pi G_{N}\right)$ as a pressure, the form of the first law appearing above identifies the mass as the enthalpy of spacetime, rather than the internal energy. In general $S$ and $V$ are independent quantities [36], but in certain cases (for example Reissner-Nordstrom-AdS) they both depend on a single parameter, with $S \sim V^{(D-2) /(D-1)}$. The implications of the thermodynamic volume have been extensively explored in the gravitational context—see Ref. [37] for a recent review_-but its role in holography remains comparatively unexplored (though see Refs. [38-45] for progress on this front).

There have been already a number of attempts to connect thermodynamic volume to the idea of complexity. There is a sense in which this is natural-in many situations the thermodynamic volume is related to the spacetime volume inside the black hole $[36,46]$, which is precisely what complexity is designed to probe. However, these investigations have either invoked new proposals for complexity [47,48], or reexpressed known results in terms of the thermodynamic volume for interpretational reasons $[22,49,50]$. Our result is the first to show concretely that thermodynamic volume emerges naturally and unambiguously in both the original CV and CA proposals in a way wholly distinct from entropy.

Solutions and global structure.-The Myers-Perry-AdS black hole solutions in $D=2 N+3$ odd dimensions are characterized by their mass and $N+1$ independent angular momenta $J_{i}$ [51]. In the special case where all angular momenta are equal, considerable simplification occurs. The metric depends only on the radial coordinate and the line element reads [52]

$$
\begin{aligned}
d s^{2}= & -f(r)^{2} d t^{2}+g(r)^{2} d r^{2}+h(r)^{2}[d \psi+A-\Omega(r) d t]^{2} \\
& +r^{2} \hat{g}_{a b} d x^{a} d x^{b}
\end{aligned}
$$

where

$$
\begin{aligned}
& g(r)^{2}=\left(1+\frac{r^{2}}{\ell^{2}}-\frac{2 m \Xi}{r^{2 N}}+\frac{2 m a^{2}}{r^{2 N+2}}\right)^{-1}, \\
& h(r)^{2}=r^{2}\left(1+\frac{2 m a^{2}}{r^{2 N+2}}\right), \quad \Omega(r)=\frac{2 m a}{r^{2 N} h^{2}},
\end{aligned}
$$

and

$$
f(r)=\frac{r}{g(r) h(r)}, \quad \Xi=1-\frac{a^{2}}{\ell^{2}} .
$$

The metric $\hat{g}$ is the Fubini-Study metric on $\mathbb{C P}^{N}$ with curvature normalized so that $\hat{R}_{i j}=2(N+1) \hat{g}_{i j}$ and $A$ is a 1 -form on $\mathbb{C P}^{N}$ that satisfies $d A=2 J$ where $J$ is the Kähler form. The basic example is in $D=5$, in which case $N=1$ and we have $\mathbb{C P}^{1} \cong S^{2}$ with the metric

$$
\hat{g}=\frac{1}{4}\left(d \theta^{2}+\sin ^{2} \theta d \phi^{2}\right), \quad A=\frac{1}{2} \cos \theta d \phi .
$$

The asymptotic region is obtained in the limit $r \rightarrow \infty$, where we recover the usual $\mathrm{AdS}_{2 N+3}$ metric provided we periodically identify $\psi \sim \psi+2 \pi$.

The spacetime contains a horizon at $r=r_{+}$where $r_{+}$is the largest root of $g^{-2}\left(r_{+}\right)=0$. The hypersurface $r=r_{+}$is a smooth Killing horizon with null generator

$$
\xi=\frac{\partial}{\partial t}+\Omega_{H} \frac{\partial}{\partial \psi}, \quad \Omega_{H}=\frac{2 m a}{r_{+}^{2 N+2}+2 m a^{2}} .
$$

There is also an inner Cauchy horizon at $r=r_{-}$which is the smaller of the two positive real roots of $g^{-2}(r)$.

The conserved charges corresponding to mass and angular momentum are $[51,53]$

$M=\frac{\Omega_{2 N+1} m}{4 \pi G_{N}}\left(N+\frac{1}{2}+\frac{a^{2}}{2 \ell^{2}}\right), \quad J=\frac{\Omega_{2 N+1}}{4 \pi G_{N}}(N+1) m a$,

where $\Omega_{2 N+1}=2 \pi^{N+1} / \Gamma(N+1)$ is the area of a unit $2 N+$ 1 sphere. We emphasize that the single angular momentum $J$ corresponds to equal angular momenta $J_{i}=J /(N+1)$ in each of the $N+1$-orthogonal planes of rotation. The black hole's entropy and temperature are given by 


$$
\begin{aligned}
S & =\frac{\Omega_{2 N+1} h\left(r_{+}\right) r_{+}^{2 N}}{4 G_{N}}, \\
T & =\frac{1}{2 \pi h\left(r_{+}\right)}\left[(N+1)\left(1+\frac{r_{+}^{2}}{\ell^{2}}\right)-\frac{\ell^{2} r_{+}^{2}}{\left(r_{+}^{2}-a^{2}\right) \ell^{2}-r_{+}^{2} a^{2}}\right],
\end{aligned}
$$

while the thermodynamic volume is [54]

$$
V=\frac{r_{+}^{2(N+1)} \Omega_{2 N+1}}{2(N+1)}+\frac{4 \pi a J}{(2 N+1)(N+1)} .
$$

Note in particular that the entropy and thermodynamic volume are independent functions of $r_{+}$and $r_{-}$(or $m$ and a). Within the framework of extended thermodynamics, the thermodynamic volume is conjugate to the pressure

$$
P=-\frac{\Lambda}{8 \pi G_{N}}=\frac{(N+1)(2 N+1)}{8 \pi \ell^{2} G_{N}} .
$$

These thermodynamic quantities satisfy the extended Smarr relation Eq. (1) and first law Eq. (2).

The entropy presents two different scaling regimes, depending on whether the black hole is close to extremality or close to the static limit. For large black holes these regimes are characterized by the scaling

$$
S_{\substack{r_{+} \rightarrow 0 \\ r_{+}}}^{\sim}\left(\frac{r_{+}}{\ell}\right)^{2 N+1} \text { and } S_{\frac{r_{-} \rightarrow 1}{r_{+}}}^{\sim}\left(\frac{r_{+}}{\ell}\right)^{2 N+2} .
$$

This should be contrasted with the scaling of $V$ in the same regimes, which satisfies

$$
V_{\frac{r_{-}}{r_{+}} \sim 0}^{\sim}\left(\frac{r_{+}}{\ell}\right)^{2 N+2} \text { and } V_{\frac{r_{-} \rightarrow 1}{r_{+}} \sim}^{\sim}\left(\frac{r_{+}}{\ell}\right)^{2 N+4} .
$$

In the static limit $r_{-} / r_{+} \rightarrow 0$ the scaling of the entropy and volume is related by $S \sim V^{(D-2) /(D-1)}$, the same relationship that holds generally for the Schwarzschild-AdS and Riessner-Nordström-AdS black holes.

Because of the enhanced symmetry of the equal-spinning solution, its causal structure is qualitatively similar to that of the Riessner-Nordström-AdS solution, as can be confirmed by an analysis of the light cone structure [55,56]. Unlike the general situation for rotating black holes where $r=0$ represents a "ring singularity" that can be traversed, the timelike surface $r=0$ in these metrics is totally singular. We show in Fig. 1 a Penrose diagram for the spacetime, including also the WDW patch.

Complexity equals volume.-Let us consider now the complexity of formation within the $\mathrm{CV}$ proposal. According to the CV proposal, the complexity of a holographic state at the boundary time slice $\Upsilon$ is related to the volume of an extremal codimension-one slice $\mathcal{B}$ by

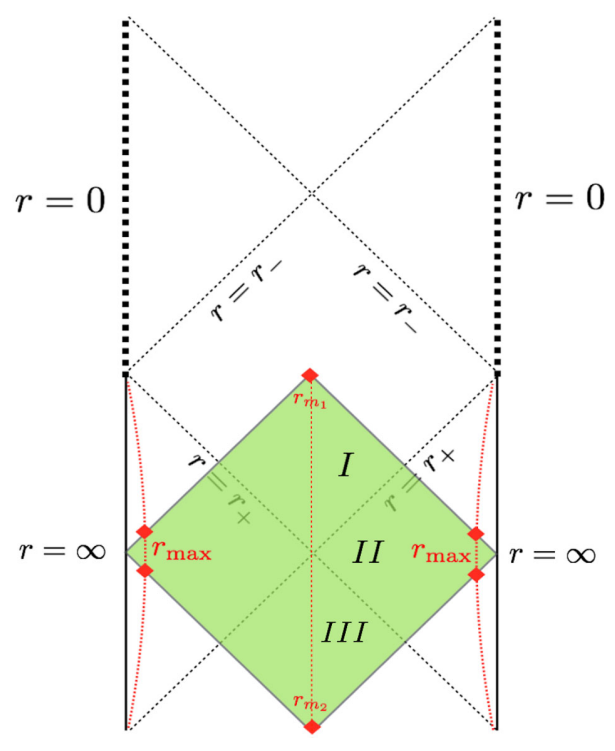

FIG. 1. A Penrose diagram for the equal-spinning Myers-PerryAdS spacetime. The shaded green region represents the WDW patch. The full diagram is an infinite strip comprised of an infinite repetition of the segment shown here.

$$
\mathcal{C}_{\mathcal{V}}(\Upsilon)=\max _{\Upsilon=\partial \mathcal{B}}\left[\frac{\mathcal{V}(\mathcal{B})}{G_{N} R}\right],
$$

where $R$ is an arbitrary length scale. Since we are interested in the complexity of formation, we consider the $t=0$ time slice of the boundary and subtract from this the analogous result for the AdS vacuum.

We take as coordinates on a codimension-one surface $(\lambda, \vec{\Omega})$ where $\vec{\Omega}$ denotes the angular coordinates of the metric. Writing the metric in ingoing coordinates $(r, v)$ and parameterizing $r=r(\lambda)$ and $v=v(\lambda)$ [57], it is straightforward to show that the volume functional is

$\mathcal{V}=2 \Omega_{D-2} \int d \lambda h(r) r^{D-3} \sqrt{-f(r)^{2} \dot{v}^{2}+2 g(r) f(r) \dot{v} \dot{r}}$.

Stationary points of this functional represent surfaces of extremal volume, while the volume of those surfaces is then obtained by evaluating Eq. (15) on shell. Straight-forward computations [58] allow us to deduce that

$$
\mathcal{V}=2 \Omega_{D-2} \int_{r_{+}}^{r_{\max }} d r r^{(D-3)} h(r) g(r),
$$

for the $t=0$ time slice. Here the integration is cutoff at some large but finite value $r_{\max }$. The complexity of formation is obtained by subtracting from Eq. (16) the analogous volume for two copies of the AdS vacuum [59]:

$$
\mathcal{V}_{\text {AdS }}=\Omega_{D-2} \int_{0}^{r_{\max }} d r \frac{r^{D-2}}{\sqrt{1+r^{2} / \ell^{2}}},
$$




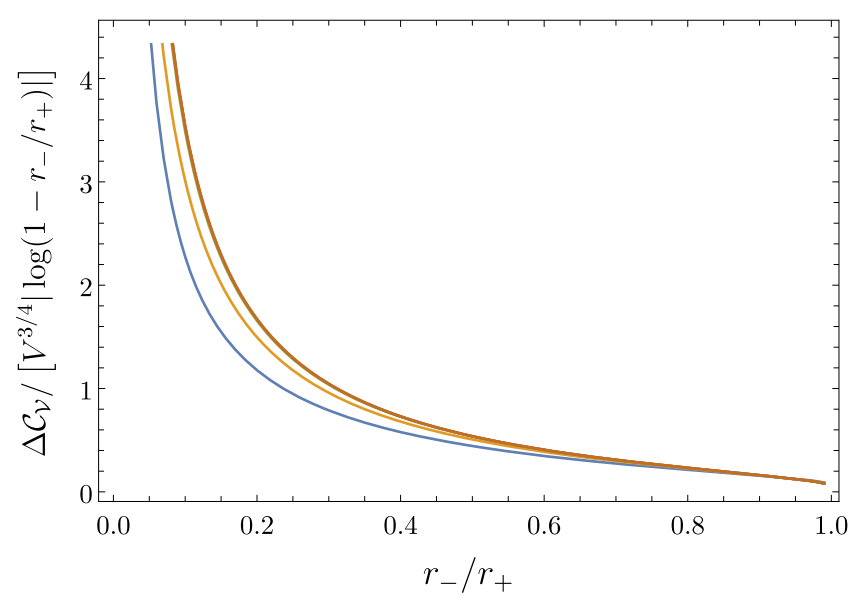

FIG. 2. A plot showing the CV complexity of formation normalized by the thermodynamic volume as a function of the ratio $r_{-} / r_{+}$in five dimensions. The plot shows curves for fixed $r_{+} / \ell=10,10^{2}, 10^{3}, 10^{4}, 10^{5}, 10^{6}$, and $10^{7}$, however after $r_{+} / \ell=1000$ the curves are visually indistinguishable.

and then taking the limit $r_{\max } \rightarrow \infty$. This yields

$$
\Delta \mathcal{C}_{\mathcal{V}}=\lim _{r_{\max } \rightarrow \infty} \frac{\left[\mathcal{V}-2 \mathcal{V}_{\mathrm{AdS}}\right]}{G_{N} R}
$$

To understand the behavior of $\Delta \mathcal{C}_{\mathcal{V}}$ for large black holes, we plot it as a function of $r_{-} / r_{+}$in Fig. 2 in $D=5$ for several different values of $r_{+} / \ell$. As extremality is approached, the complexity of formation exhibits a logarthmic divergence [58], similar to what occurs for charged black holes [23]. In the plot, we have normalized $\Delta \mathcal{C}_{\mathcal{V}}$ taking into account this divergence, and have also normalized by the thermodynamic volume to an appropriate power. While the plot shows the curve for seven distinct values of $r_{+} / \ell$, only three curves are actually distinguishable. This illustrates that, for large black holes, the scaling of the complexity of formation with $r_{+} / \ell$ matches the scaling of the thermodynamic volume.

This scaling result is not peculiar to five-dimensional black holes, but in fact holds for any (odd) dimension. To see this we have determined numerically the scaling of $\Delta \mathcal{C}_{\mathcal{V}}$ near extremality [60] in a number of higher odd dimensions, shown in Table I. In all cases we see that the scaling matches precisely that derived from $V^{(D-2) /(D-1)}$.

We thus find the intriguing result that the complexity of formation scales as $V^{(D-2) /(D-1)}$, capturing two distinct scaling behaviors in the static and near-extremal limits as in Eq. (13). Interestingly this power is the same power that relates the entropy [Eq. (9)] to thermodynamic volume [Eq. (10)] in the static solutions. However, while this means the scaling can be expressed in terms of either $S$ or $V^{(D-2) /(D-1)}$ near $r_{-} / r_{+} \rightarrow 0$, it is only the volume that captures the correct scaling behavior for all values of $r_{-} / r_{+}$- see Eqs. (12) and (13).
TABLE I. Table comparing scaling of $\Delta \mathcal{C}_{\mathcal{V}}$ with the scaling of the thermodynamic volume $V^{(D-2) /(D-1)}$ for large $r_{+} / \ell$. The $\Delta \mathcal{C}_{\mathcal{V}}$ data is obtained numerically by evaluating the complexity of formation between $r_{+} / \ell=10^{10}$ and $r_{+} / \ell=10^{20}$ and we work close to extremality with $r_{-} / r_{+}=1-10^{-10}$.

\begin{tabular}{lcc}
\hline \hline Dimension & $\beta$ such that $\Delta \mathcal{C}_{\mathcal{V}} \sim\left(r_{+} / \ell\right)^{\beta}$ & $V^{(D-2) /(D-1)}$ \\
\hline 5 & 4.50000 & $9 / 2=4.5$ \\
7 & 6.66667 & $20 / 3 \approx 6.66667$ \\
9 & 8.75000 & $35 / 4=8.75$ \\
11 & 10.80000 & $54 / 5=10.8$ \\
13 & 12.83333 & $77 / 6 \approx 12.83333$ \\
15 & 14.85714 & $104 / 7 \approx 14.85714$ \\
17 & 16.87500 & $135 / 8 \approx 16.87500$ \\
19 & 18.88889 & $170 / 9 \approx 18.88889$ \\
21 & 20.90000 & $209 / 10=20.9$ \\
23 & 22.90909 & $252 / 11 \approx 22.90909$ \\
25 & 24.91667 & $299 / 12 \approx 24.91667$ \\
27 & 26.92308 & $350 / 13 \approx 26.92308$ \\
\hline \hline
\end{tabular}

Complexity equals action.-We have now demonstrated that in the CV proposal it is the thermodynamic volume and not the entropy that characterizes the complexity of formation for large black holes. It is natural to ask whether this behavior is universal to both complexity proposals, or if it is a peculiar behavior associated with the $\mathrm{CV}$ proposal. Here we show that the same feature emerges for the CA proposal.

In the CA proposal, the complexity of the CFT state at boundary time $t$ is given by the value of the gravitational action evaluated on the WDW patch of spacetime

$$
\mathcal{C}_{\mathcal{A}}(\Upsilon)=\frac{I_{\mathrm{WDW}}}{\pi}
$$

The WDW patch is defined as the domain of dependence of the bulk Cauchy slice that intersects the boundary at the given time slice $\Upsilon$. The geometry of this patch for the rotating black holes is shown in Fig. 1. There are a number of nontrivial contributions to the action arising in this computation, including joint contributions at the future and past meeting points of the null sheets of the WDW patch, joint and boundary terms at the regularization of the patch near infinity, and a null boundary counterterm along the null sheets of the WDW patch. A full account of these terms will be presented elsewhere [58], but it suffices to say that the computation is morally similar to the case of charged black holes [23].

The result of this analysis is that the complexity of formation in the CA proposal is given by

$$
\Delta \mathcal{C}_{\mathcal{A}}=\frac{I_{\mathrm{WDW}}-2 I_{\mathrm{AdS}}}{\pi}
$$

with 


$$
\begin{aligned}
\pi \Delta \mathcal{C}_{\mathcal{A}}= & \frac{\Lambda \Omega_{2 N+1}}{2(N+1)(2 N+1) \pi G_{N}}\left[\int_{r_{m_{0}}}^{\infty} d r r^{2 N+1}\left(g(r)^{2} h(r)-\frac{r}{1+r^{2} / \ell^{2}}\right)-\int_{0}^{r_{m_{0}}} d r \frac{r^{2(N+1)}}{1+r^{2} / \ell^{2}}\right]-\frac{\Omega_{2 N+1}\left(r_{m_{0}}\right)^{2 N+1}}{2 \pi G_{N}(2 N+1)} \\
& -\frac{\Omega_{2 N+1}}{4 \pi G_{N}}\left(r_{m_{0}}\right)^{2 N} h\left(r_{m_{0}}\right) \log \ell_{\mathrm{ct}}^{2} \Theta\left(r_{m_{0}}\right)^{2}\left|f\left(r_{m_{0}}\right)^{2}\right|-\frac{\Omega_{2 N+1}}{2 \pi G_{N}} \int_{r_{m_{0}}}^{\infty} d r r^{2 N}\left[h(r) \frac{\Theta^{\prime}}{\Theta}+1\right]
\end{aligned}
$$

where

$$
\Theta=\frac{1}{f(r) g(r)}\left[\frac{2 N}{r}+\frac{h^{\prime}}{h}\right]
$$

Here the constant $\ell_{\mathrm{ct}}$ comes from a counterterm on the null boundaries. Such a term is not required for a well-posed variational problem, but is required to ensure the final result does not depend on the parameterization of the null generators of the WDW patch [61], and moreover has been shown to be important for reproducing certain required properties of complexity in some situations $[16,25,62,63]$. The parameter $r_{m_{0}}$ is the value of $r$ at which the future and past tips of the WDW patch meet. It is determined by solving the equation $r^{*}\left(r_{m_{0}}\right)=0$ where

$$
r^{*}(r)=\int_{\infty}^{r} \frac{g^{2}(\tilde{r}) h(\tilde{r})}{\tilde{r}} d \tilde{r}
$$

is the tortoise coordinate.

The most difficult part of the CA computation is the determination of $r_{m_{0}}$. In some instances, particularly in the limit $r_{-} / r_{+} \rightarrow 0$, accurate determination of this parameter requires hundreds of digits of precision in the numerics. This technicality has limited our ability to probe the behavior of the complexity of formation within the CA

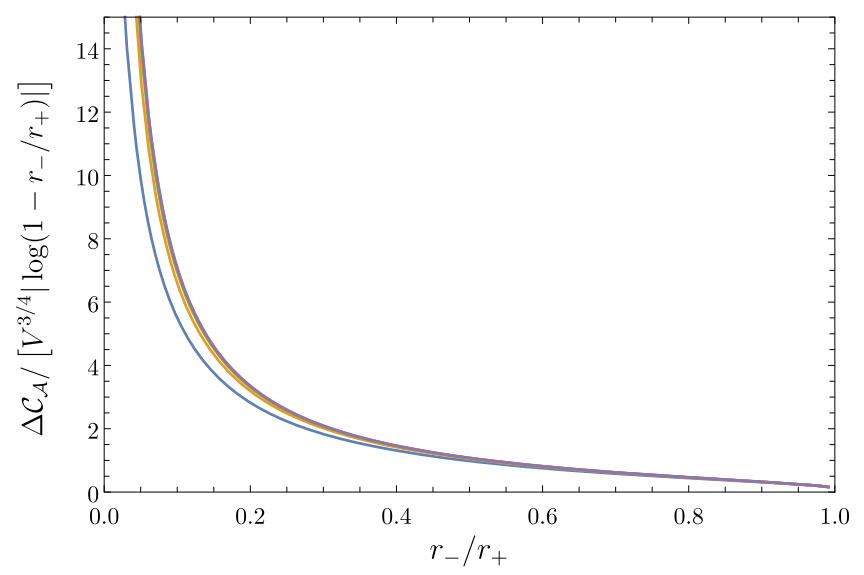

FIG. 3. A plot showing the CA complexity of formation normalized by the thermodynamic volume as a function of the ratio $r_{-} / r_{+}$in five dimensions. The plot shows curves for fixed $r_{+} / \ell=10,10^{2}, 10^{3}, 10^{4}, 10^{5}, 10^{6}$, and $10^{7}$, however after $r_{+} / \ell=1000$ the curves are visually indistinguishable. Here we have set $\ell_{\mathrm{ct}}=\ell$. conjecture as broadly as the CV conjecture. However, we show in Fig. 3 the result of the action computation in five dimensions. The plot makes clear that the thermodynamic volume controls the scaling of $\Delta \mathcal{C}_{\mathcal{A}}$ for large black holes, just as in the $\mathrm{CV}$ conjecture. While it was possible to compute the behavior in various higher dimensions for the $\mathrm{CV}$ case, this is more difficult in the CA scenario. Nonetheless, we have confirmed the scaling with thermodynamic volume in seven dimensions, which suggests the same trend holds in general for CA.

Discussion.-We have shown here for the first time that the thermodynamic volume plays a natural role in both the $\mathrm{CA}$ and $\mathrm{CV}$ conjectures. Reinstating units, the complexity of formation of large black holes obeys the same scaling as the thermodynamic volume

$$
\Delta \mathcal{C}=\Sigma_{g} C_{T}\left(\frac{V}{V_{\mathrm{AdS}}}\right)^{\frac{D-2}{D-1}}
$$

where $V_{\text {AdS }}=\ell^{D-1}, \Sigma_{g}$ is a factor that depends on the specific metric, dimension, etc. but not on the size of the black hole, and $C_{T} \sim \ell^{D-2} / G_{N}$ is the central charge of the CFT.

This proposal reproduces known results for static black holes, as in those cases the thermodynamic volume is not independent from the entropy, $S \sim V^{(D-2) /(D-1)}$, and the above can be recast in terms of the entropy in those cases. However, for rotating black holes the volume and entropy are independent and it becomes clear that it is Eq. (24) that captures the correct behavior, and not an analogous expression involving the entropy. Our result also reproduces the behavior of the complexity of formation for gravitational solitons [20], which are horizonless geometries that possess thermodynamic volume but no entropy.

The thermodynamic volume has been conjectured [36] to obey a "reverse" isoperimetric inequality:

$$
\mathcal{R} \equiv\left(\frac{(D-1) V}{\Omega_{D-2}}\right)^{1 /(D-1)}\left(\frac{\Omega_{D-2}}{4 G_{N} S}\right)^{1 /(D-2)} \geq 1 .
$$

The inequality is saturated by (charged) SchwarzschildAdS spacetimes. Assuming the relationship [Eq. (24)] is general, the reverse isoperimetric inequality becomes the statement

$$
\Delta \mathcal{C} \geq \beta_{D} S
$$


where $\beta_{D}$ is a positive constant that can be easily worked out from the above. This means that the complexity of formation for large black holes is bounded from below by the entropy (equivalently, the number of degrees of freedom).

What we have said so far concerns the complexity of formation. Before closing, let us remark that there is also a connection between the late time growth of complexity and thermodynamic volume, again for large black holes. For the rotating black holes considered here this relationship works out to be [58]

$$
\dot{\mathcal{C}}=N_{\mathcal{A}, \mathcal{V}} P \Delta V
$$

where $\Delta V$ is the difference between the thermodynamic volumes of the inner and outer horizons and $N_{\mathcal{A}, \mathcal{V}}$ is a proportionality constant whose numeric value depends on whether one uses the $\mathrm{CV}$ or CA conjecture. The implication of this is that not only does the thermodynamic volume control the complexity of formation, but we see here that it also controls the late-time growth.

Our results for the complexity of formation draw a clear and simple connection between thermodynamic volume and holographic complexity. A better understanding of complexity in the holographic dictionary would then lead to a simple and direct holographic interpretation of thermodynamic volume and vice versa. Going forward, it will be important to assess the validity of our proposal, Eq. (24), as broadly as possible. Exploring the properties of complexity of formation in other spacetimes where $S$ and $V$ are independent would contribute additional evidence toward the generality of the relationship, or could constitute a counterexample from which its possible limitations could be assessed.

We thank Hugo Marrochio for useful discussions. This work was supported in part by the Natural Sciences and Engineering Research Council of Canada. The work of R. A. H. is supported by the Natural Sciences and Engineering Research Council of Canada through the Banting Postdoctoral Fellowship program. H. K. K. acknowledges the support of NSERC Discovery Grant No. RGPIN-2018-04887.

*a2albalu@uwaterloo.ca

†rhennigar@mun.ca

thkkunduri@mun.ca

§rbmann@uwaterloo.ca

[1] S. Ryu and T. Takayanagi, J. High Energy Phys. 08 (2006) 045.

[2] V. E. Hubeny, M. Rangamani, and T. Takayanagi, J. High Energy Phys. 07 (2007) 062.

[3] H. Casini, M. Huerta, and R. C. Myers, J. High Energy Phys. 05 (2011) 036.

[4] A. Lewkowycz and J. Maldacena, J. High Energy Phys. 08 (2013) 090.
[5] L. Susskind, Fortschr. Phys. 64, 24 (2016); 64, 44(A) (2016).

[6] L. Susskind, Fortschr. Phys. 64, 49 (2016).

[7] S. Chapman, M. P. Heller, H. Marrochio, and F. Pastawski, Phys. Rev. Lett. 120, 121602 (2018).

[8] R. A. Jefferson and R. C. Myers, J. High Energy Phys. 10 (2017) 107.

[9] D. Stanford and L. Susskind, Phys. Rev. D 90, 126007 (2014).

[10] A. R. Brown, D. A. Roberts, L. Susskind, B. Swingle, and Y. Zhao, Phys. Rev. Lett. 116, 191301 (2016).

[11] A. R. Brown, D. A. Roberts, L. Susskind, B. Swingle, and Y. Zhao, Phys. Rev. D 93, 086006 (2016).

[12] See, e.g., Refs. [13,14] for preliminary investigations into the connections between the circuit complexity and holographic complexity proposals.

[13] A. Bernamonti, F. Galli, J. Hernandez, R. C. Myers, S.-M. Ruan, and J. Simón, Phys. Rev. Lett. 123, 081601 (2019).

[14] A. Bernamonti, F. Galli, J. Hernandez, R. C. Myers, S.-M. Ruan, and J. Simón, J. Phys. A 53, 294002 (2020).

[15] D. Carmi, R. C. Myers, and P. Rath, J. High Energy Phys. 03 (2017) 118.

[16] S. Chapman, H. Marrochio, and R. C. Myers, J. High Energy Phys. 06 (2018) 114.

[17] Z.-Y. Fan and M. Guo, Nucl. Phys. B950, 114818 (2020).

[18] S. Chapman, D. Ge, and G. Policastro, J. High Energy Phys. 05 (2019) 049.

[19] A. Ghosh and R. Mishra, arXiv:1907.11757.

[20] S. Andrews, R. A. Hennigar, and H. K. Kunduri, Classical Quantum Gravity 37, 204002 (2020).

[21] R. G. Cai, S. M. Ruan, S. J. Wang, R. Q. Yang, and R. H. Peng, J. High Energy Phys. 09 (2016) 161.

[22] H. Huang, X.-H. Feng, and H. Lu, Phys. Lett. B 769, 357 (2017).

[23] D. Carmi, S. Chapman, H. Marrochio, R. C. Myers, and S. Sugishita, J. High Energy Phys. 11 (2017) 188.

[24] P. A. Cano, R. A. Hennigar, and H. Marrochio, Phys. Rev. Lett. 121, 121602 (2018).

[25] S. Chapman, H. Marrochio, and R. C. Myers, J. High Energy Phys. 06 (2018) 046.

[26] S. W. Hawking, C. J. Hunter, and M. M. Taylor-Robinson, Phys. Rev. D 59, 064005 (1999).

[27] S. W. Hawking and H. S. Reall, Phys. Rev. D 61, 024014 (1999).

[28] M. M. Caldarelli, G. Cognola, and D. Klemm, Classical Quantum Gravity 17, 399 (2000).

[29] M. Brigante, H. Liu, R. C. Myers, S. Shenker, and S. Yaida, Phys. Rev. D 77, 126006 (2008).

[30] R. C. Myers, M. F. Paulos, and A. Sinha, J. High Energy Phys. 08 (2010) 035.

[31] R. C. Myers and A. Sinha, J. High Energy Phys. 01 (2011) 125.

[32] M. Mezei, Phys. Rev. D 91, 045038 (2015).

[33] P. Bueno, R. C. Myers, and W. Witczak-Krempa, Phys. Rev. Lett. 115, 021602 (2015).

[34] P. Bueno, P. A. Cano, R. A. Hennigar, and R. B. Mann, Phys. Rev. Lett. 122, 071602 (2019).

[35] D. Kastor, S. Ray, and J. Traschen, Classical Quantum Gravity 26, 195011 (2009).

[36] M. Cvetic, G. W. Gibbons, D. Kubiznak, and C. N. Pope, Phys. Rev. D 84, 024037 (2011). 
[37] D. Kubiznak, R. B. Mann, and M. Teo, Classical Quantum Gravity 34, 063001 (2017).

[38] C. V. Johnson, Classical Quantum Gravity 31, 205002 (2014).

[39] D. Kastor, S. Ray, and J. Traschen, J. High Energy Phys. 11 (2014) 120.

[40] A. Karch and B. Robinson, J. High Energy Phys. 12 (2015) 073.

[41] E. Caceres, P. H. Nguyen, and J. F. Pedraza, Phys. Rev. D 95, 106015 (2017).

[42] M. Sinamuli and R. B. Mann, Phys. Rev. D 96, 086008 (2017).

[43] C. V. Johnson and F. Rosso, Classical Quantum Gravity 36, 015019 (2019).

[44] C. V. Johnson, V. L. Martin, and A. Svesko, Phys. Rev. D 101, 086006 (2020).

[45] F. Rosso and A. Svesko, J. High Energy Phys. 08 (2020) 008.

[46] A. B. Bordo, J. High Energy Phys. 12 (2020) 116.

[47] J. Couch, W. Fischler, and P. H. Nguyen, J. High Energy Phys. 03 (2017) 119.

[48] Z.-Y. Fan and M. Guo, J. High Energy Phys. 08 (2018) 031; 09 (2019) 121(E).

[49] H.-S. Liu, H. Lü, L. Ma, and W.-D. Tan, J. High Energy Phys. 07 (2020) 090.

[50] W. Sun and X.-H. Ge, arXiv:1912.00153.

[51] G. Gibbons, H. Lu, D. N. Page, and C. Pope, J. Geom. Phys. 53, 49 (2005).

[52] H. K. Kunduri, J. Lucietti, and H. S. Reall, Phys. Rev. D 74, 084021 (2006).

[53] G. Gibbons, M. Perry, and C. Pope, Classical Quantum Gravity 22, 1503 (2005).
[54] N. Altamirano, D. Kubiznak, R. B. Mann, and Z. Sherkatghanad, Galaxies 2, 89 (2014).

[55] F. Pretorius and W. Israel, Classical Quantum Gravity 15, 2289 (1998).

[56] A. A. Balushi and R. B. Mann, Classical Quantum Gravity 36, 245017 (2019).

[57] This choice is possible only because of the enhanced symmetry of the metric. For a general rotating black hole these functions would depend also on the polar angles.

[58] A. Al Balushi, R. A. Hennigar, H. K. Kunduri, and R. B. Mann, arXiv:2010.11203.

[59] It is straightforward to show [58] that differences in the definition of $r_{\max }$ between the black hole spacetime and global AdS do not contribute to the integral.

[60] In the static limit, $S$ and $V^{(D-2) /(D-1)}$ behave in the same manner, so it is only the extremal limit that distiniguishes entropy from volume.

[61] L. Lehner, R. C. Myers, E. Poisson, and R. D. Sorkin, Phys. Rev. D 94, 084046 (2016).

[62] C. A. Agón, M. Headrick, and B. Swingle, J. High Energy Phys. 02 (2019) 145.

[63] M. Alishahiha, K. Babaei Velni, and M. R. Mohammadi Mozaffar, Phys. Rev. D 99, 126016 (2019).

[64] See Supplemental Material at http://link.aps.org/ supplemental/10.1103/PhysRevLett.126.101601 for additional details on the determination of the scaling of the complexity of formation as a function of black hole size, as well as a comparison with the scaling of the black hole entropy. 